\title{
Was ist empirische Ethik?
}

\author{
Bert (A.W.) Musschenga
}

Online publiziert: 29. August 2009

(C) Die Autor(en) 2009. Dieser Artikel ist auf Springerlink.com mit Open Access verfügbar.

Zusammenfassung Empirische Ethik ist ein relativ neues Vorgehen in der Ethikforschung, das vor allem in der Medizinethik angewandt wird. Dieser Beitrag bespricht die kennzeichnenden Charakteristika der empirischen Ethik und unterscheidet zwischen generalistischer und kontextualistischer empirischer Ethik. Zuerst werden verschiedene Beispiele beider Arten von empirischer Ethik vorgestellt, danach werden für beide Ansätze mögliche Schwachpunkte diskutiert. Die Schlussfolgerung des Beitrages besteht darin, dass das Entstehen der empirischen Ethik eine positive Entwicklung ist. Empirische Ethik sollte jedoch als eine Ergänzung der traditionellen philosophischen Medizinethik betrachtet werden, und nicht als die bessere Alternative.

Schlüsselwörter (Generalistische, kohärentistische, kontextualistische) empirische Ethik $\cdot$ Kontextsensitivität $\cdot$ Medizinanthropologie $\cdot$ Medizinethik $\cdot$ Medizinsoziologie Patientenautonomie

\section{What is empirical ethics?}

Abstract Definition of the problem Empirical ethics is a relatively new type of research, predominantly practiced in medical ethics. Arguments This article discusses the distinctive features of empirical ethics and makes a distinction between generalist and contextualist empirical ethics. After providing some examples of both types of empirical ethics, it discusses a weak spot in each of them. Conclusion The conclusion is that the emergence of empirical ethics as such is a positive development. Empirical ethics, however, should be regarded as a complement to 'traditional' philosophical medical ethics, and not as the better alternative.

B. (A.W.) Musschenga ( $\square$ )

Philosophische Fakultät, Vrije Universiteit, De Boelelaan 1105

1081 HV Amsterdam, Niederlande

E-Mail: aw.musschenga@ph.vu.nl 
Keywords Competence $\cdot$ Context-sensitivity $\cdot($ Generalist, coherentist, contextualist) empirical ethics $\cdot$ Medical anthropology $\cdot$ Medical ethics $\cdot$ Medical sociology $\cdot$ Patient autonomy

Die Ethik wird meist in deskriptive Ethik, normative Ethik und Meta-Ethik unterteilt. Die deskriptive Ethik beschreibt die Moral, d.h. die moralischen Ideale, Werte, Prinzipien, Regeln, Tugenden einer Gruppe; die normative Ethik schreibt vor, was ein gutes und gelingendes Leben und richtiges Handeln ist, und die Meta-Ethik analysiert die Bedeutung und Funktion moralischer Begriffe und behandelt die Frage, ob und wie moralische Urteile begründet werden können. Seit den Neunzigerjahren des vorigen Jahrhunderts erschienen vor allem in den Niederlanden und in Großbritannien immer mehr Publikationen, in denen Forscher, meist aus dem Fachbereich der Medizin und des Gesundheitswesens kommend, ihre Arbeiten als empirisch-ethische Forschung bezeichnen. Außerdem erschienen auch Artikel, in denen die Methode und das Charakteristische der empirischen Ethik näher zu definieren versucht wurde. ${ }^{1}$ In den Niederlanden scheint die empirische Ethik im Moment dabei zu sein, zur Standardmethode der medizinischen Ethik zu werden.

In diesem Artikel möchte ich im Folgenden zuerst die wesentlichen Merkmale der empirischen Ethik beschreiben. Im Anschluss werde ich die Motive sowie deren Zielsetzungen der empirischen Ethik besprechen. Hierbei unterscheide ich dann zwischen zwei Hauptrichtungen der empirischen Ethik, der generalistischen und der kontextualistischen empirischen Ethik, und werde hierfür einige Beispiele angeben. Im vorletzten Abschnitt des Artikels bespreche ich kritische Aspekte sowohl der generalistischen als auch der kontextualistischen Richtung und schließe mit einer kurzen Bewertung.

\section{Einordnung und Beschreibung der empirischen Ethik}

Der belgische Medizinethiker Pascal Borry und seine Kollegen fanden in älteren Jahrgängen von Ethics zwei Artikel, in denen der Begriff empirische Ethik im Titel vorkommt [4]. ${ }^{2}$ Die Autoren dieser Artikel scheinen unter empirischer Ethik eine Form des Empirismus zu verstehen, in dem Werte auf Bedürfnisse zurückgeführt werden. Bereits in den Fünfzigerjahren des vorigen Jahrhunderts gab es einen Ansatz, welcher der Ethik ein wissenschaftliches Fundament geben wollte. Vertreter dieser Richtung waren u. a. der flämische Philosoph Jacques Kruithof und der amerikanische Philosoph Abraham Edel. Kruithof verwendete in seinem Buch Eticologie. Inleiding tot de studie van het morele verschijnsel [Ethikologie. Einführung in das Studium des Phänomens Moral] [17] hauptsächlich Erkenntnisse der Psychologie. Edel schöpfte in seinem Ethical Judgement. The Use of Science in Ethics [9] aus einem breiteren Spektrum von Wissenschaften: sowohl aus der Psychologie, der Biologie, der Soziologie, der Kulturanthropologie als auch aus den Geschichtswissenschaften. Auf diese Weise versuchte er, das Problem des ethischen Relativismus in Angriff zu nehmen und die Unbestimmtheit ethischer Urteile zu vermindern. In seinem Science and the

\footnotetext{
${ }^{1}$ Für einen Überblick über die empirische Ethik auf dem Gebiet der Medizin und des Gesundheitswesens verweise ich auf zwei Themenhefte von Zeitschriften: Bd. 7, Nr. 1 von Medicine, Health Care and Philosophy und Bd. 23, Nr. 4 von Bioethics, sowie auf den Sammelband von Guy Widdershoven et al. [38]. Beide Zeitschriften, aber insbesondere Medicine, Health Care and Philosophy publizieren auch in den regulären Nummern regelmäßig Artikel über empirische Ethik.

${ }^{2}$ Eliseo Vivas [36], James K. Feibleman [11].
} 
Structure of Ethics [10] entwickelte er sogar ein Modell zur Verbindung der empirischen Wissenschaften mit der Ethik. Zu der Forschungsrichtung, zu der Kruithof und Edel gehören, kann man auch die amerikanische „Society for Empirical Ethics“ zählen, welche zum Ziel hat, interdisziplinäre Diskussionen zwischen Philosophen und Sozialwissenschaftlern zu fördern, die an einer empirisch fundierten Definition des aristotelischen Begriffs der Eudaimonia ${ }^{3}$ arbeiten wollen. Des Weiteren gibt es derzeit insbesondere in den Vereinigten Staaten eine Gruppe von Philosophen, (Sozial)Psychologen und Kognitions- und Neurowissenschaftlern, die auf einem Gebiet forschen, das der Philosoph John Doris [8] als ,empirisch informierte Ethik“ bezeichnete. ${ }^{4}$ Hierbei geht es um Forschungsansätze, in denen man empirische Annahmen in ethischen Theorien mit Ergebnissen empirischer Studien konfrontiert, um die Plausibilität ethischer Theorien zu prüfen und ein neues Licht auf seit langem bestehende Kontroversen in der ethischen Theorie zu werfen.

In der empirischen Ethik im Kontext von Medizin und Gesundheitswesen meint empirisch faktisch sozialwissenschaftliche empirische Forschung. Der Begriff empirische Ethik verweist somit auf eine Kombination von Sozialwissenschaften und normativer Ethik. Es ist allerdings nicht ungewöhnlich, dass Ethiker Sozialwissenschaften oder eine andere empirische Wissenschaft verwenden, um Einblicke in den Problembereich zu erhalten, auf den ihre ethischen Reflexionen zielen. In welcher Hinsicht unterscheidet sich somit die empirische Ethik von dieser Art von Forschung, so dass es als notwendig erachtet wird, ihr einen eigenen Namen zu geben? Die am ehesten zufriedenstellende Antwort auf diese Frage geben John McMillan und Tony Hope. Empirische Ethik ist ihrer Meinung nach mehr als nur das Sammeln von Daten, die anschließend einer ethischen Analyse unterworfen werden, oder auch als eine ethische Analyse, auf deren Grundlage Daten gesammelt werden. Nur Untersuchungen, die ein zyklisches Modell verwendeten, in dem ethische Analyse und empirische Datensammlung einander in einem interaktiven Zyklus informierten, könnten als ,empirische Ethik“" bezeichnet werden ([19], S. 19). ${ }^{5}$

Ich habe nicht überprüft, ob alle Studien, die von den Autoren selbst als empirische Ethik bezeichnet werden, dem Kriterium von McMillan und Hope genügen, es ist jedoch ein gut nachvollziehbares Kriterium. Wie bereits festgestellt, beschränkt sich die empirische Forschung innerhalb der empirischen Ethik faktisch auf sozialwissenschaftliche Untersuchungen, dies muss jedoch nicht notwendigerweise so sein. Die meisten Untersuchungen, die als empirische Ethik bezeichnet werden, finden sich in Medizin und Gesundheitswesen sowie ein kleiner Teil auf dem Gebiet der Wirtschaftsethik. ${ }^{6}$ Auch diese Einschränkung erscheint mir eher zufällig. Vergleichbare Versuche, sozialwissenschaftliche Untersuchungen mit philosophischer Analyse zu verbinden, finden wir auch in der politischen Philosophie in Bezug auf soziale Gerechtigkeit. Philosophen versuchen, eine Verbindung herzustellen zwischen ihrer normativen Reflexion über soziale Gerechtigkeit und der sozialwissenschaftlichen social justice research, jedoch bezeichnen sie ihre Untersuchungen nicht als ,empirische Ethik“". Wie bereits erwähnt, beschränke ich mich im Folgenden auf die empirische Ethik im Kontext von Medizin und Gesundheitswesen. Wenn ich daher über empirische Ethik

\footnotetext{
${ }_{3}^{3}$ Europäische Philosophen, die an ähnlichen Programmen arbeiten, sind Philippa Foot [12] und Malcolm Parker [25].

${ }^{4}$ Für das Programm der empirisch informierten Ethik siehe auch Stephen Stich und John Doris [31].

5 Siehe auch die Ideen von Bert Molewijk und Mitarbeitern über eine ,integrierte empirische Ethik“ [21].

${ }^{6}$ Die Beziehung zwischen normativer Ethik und Business-Ethics wird bei Weaver und Trevino [37] besprochen.

7 Siehe u. a. Volkert Schmidt [30] und die von Schmidt redigierte Themennummer von Ethical Theory and Moral Practice, Bd. 2, Nr. 4.
} 
spreche, meine ich die empirische Medizinethik. Empirische Ethik ist somit eine integrierte Kombination von ethischer Analyse und empirischer Forschung. Eine Voraussetzung für die empirische Ethik scheint mir zu sein, dass Datensammlung und ethische Analyse in einer Studie von derselben Forschergruppe durchgeführt werden. Hierdurch ist es möglich, einen interaktiven Zyklus von ethischer Analyse und Datensammlung zu schaffen. Wo ist jedoch die empirische Ethik innerhalb der vorher genannten Dreiteilung der Ethik anzusiedeln? Im Hinblick auf ihre Zielsetzungen ist die empirische Ethik eine normative Forschung, welche die Methoden der philosophischen normativen Ethik und der (sozialwissenschaftlichen) empirischen Forschung verwendet.

\section{Warum und wozu empirische Ethik?}

Die empirische Ethik versteht sich als Antwort sowohl auf die externe Kritik der Sozialwissenschaftler als auch auf die interne Kritik der Ethiker an der gängigen (medizinischen) Ethik. Ich beginne mit der externen Kritik, welche vor allem von Medizinsoziologen formuliert wurde. Der amerikanische Soziologe Robert Zussman, der umfangreiche Feldstudien auf den Intensivstationen zweier amerikanischer Krankenhäusern durchgeführt hat, stellte fest, dass Medizinethiker durch den Fokus auf die Frage, wie Entscheidungen getroffen werden sollen, sich bemerkenswert wenig darum kümmerten, wie diese tatsächlich getroffen werden. "As a result, medical ethics, and consequently the public policies that medical ethics have helped to shape, have been characterized by striking omissions" ([40], S. 2). Die medizinische Ethik hat nach Zussman also den sozialen Kontext, in dem medizinische Entscheidungen getroffen werden, konsequent vernachlässigt. Er verweist dabei auf seinen Kollegen Renee Fox [13], der feststellte, dass Medizinethiker medizinische Entscheidungen häufig so konzeptualisieren, als ob sie auf Verträgen beruhten - also auf rationalen und freiwilligen Vereinbarungen zwischen unabhängigen Individuen, meist zwischen einem Arzt und einem Patienten. Medizinethiker würden die Bedeutung formaler Verfahren zum Erhalt „informierter Zustimmung“ betonen, sie sähen jedoch über die sozialen Kräfte, welche die Werte des Patienten beeinflussten, über die Asymmetrie zwischen Arzt und Patient und über nicht-rationale Faktoren hinweg, welche die Bereitschaft des Patienten beeinflussten, einer Behandlung zuzustimmen ([13], S. 2). Ein Teil der Untersuchungen darüber, wie Entscheidungen tatsächlich zustande kämen, müsse jene Werte berücksichtigen, die den Entscheidungen zugrunde liegen, und beachten, "how those values articulate with other values, how they emerge from the interests and influence of different groups and organizations, and what in the organization of medicine or American society frustrates the realization of other values" ([40], S. 3).

Zussman fordert mit seiner Unterscheidung zwischen „how decisions should be made“ und ,how they are in fact made" nicht nur die Berücksichtigung der faktischen Merkmale von Entscheidungen und der Schwierigkeiten bei der Umsetzung der Werte und Prinzipien in der Praxis. Er verwendet sie auch, um auf einen Konflikt zwischen der Werteperspektive der medizinischen Ethik als soziale Bewegung und der etablierten medizinischen Praxis hinzuweisen. Er vertritt die Meinung, dass die Medizinethiker sich nur unzureichend bewusst sind, dass sie ein eigenständiges Set von Werten und Interessen fordern, wie etwa die Selbstbestimmung, welche der medizinischen Tradition jedoch fremd ist. Die Tatsache, dass die Medizin "has a long history of turning to its own purposes the attempts of others to regulate $i t$ " sei Grund genug zu untersuchen, wie die medizinische Praxis mit den Werten der medizinischen Ethik umgehe. Darum ist seiner Meinung nach eine Soziologie der medizinischen Ethik ebenso notwendig wie eine Philosophie der medizinischen Ethik ([40], S.3). 
Die Feststellung, dass Ethiker häufig den sozialen Kontext medizinischer Entscheidungen nur unzureichend berücksichtigen, findet sich in vielen anderen Publikationen über die Beziehung zwischen Sozialwissenschaften und Ethik wieder. Robert Pool, ein niederländischer Medizinanthropologe, der die Euthanasiepraxis in einem niederländischen Krankenhaus untersuchte, sieht ebenfalls Diskrepanzen zwischen der Sicht der medizinischen Ethik hinsichtlich Euthanasieentscheidungen und dem tatsächlichen Gang der Dinge. Er behauptet, dass Medizinethiker anscheinend denken, derartige Entscheidungen würden zu einem eindeutig feststellbaren Zeitpunkt von einer einzelnen Person getroffen. Tatsächlich hätten aber Euthanasieentscheidungen Prozesscharakter - ein Prozess, in dem verschiedene Akteure eine Rolle spielten: die Patienten, ihre Familien, Ärzte und das Pflegepersonal ([27], S. 201 f.). Dieser Prozess würde auch durch gesetzliche Vorschriften beeinflusst sowie durch spezifische Protokolle und Verfahrensweisen oder durch das Krankenhaus. Anne-Mei The, ebenfalls eine niederländische Medizinanthropologin, die das Dilemma der Pflegenden bei Entscheidungen in terminalen Fällen untersuchte, kommt zu einer ähnlichen Schlussfolgerung. Derartige Entscheidungen werden ihrer Meinung nach nicht individuell, sondern kollektiv getroffen und haben Prozesscharakter. Sie seien das Ergebnis eines Vorgangs, der als Prozess des Wachsens und des Reifens beschrieben werden könne [33]. ${ }^{8}$

Soweit also die externe Kritik der Sozialwissenschaftler. Sie zielt darauf ab, dass Ethiker vor allem den prozessualen und prozeduralen Aspekten ethischer Entscheidungen in Krankenhäusern und Pflegeheimen mehr Aufmerksamkeit schenken. Die internen Kritiker - die Kritiker aus der medizinischen Ethik selbst - werfen der gängigen medizinischen Ethik vor, dass sie zu abstrakt, zu allgemein sowie unrealistisch und nicht durchführbar sei und den einzigartigen Merkmalen spezifischer Situationen nicht gerecht würde. Die gängige medizinische Ethik hat sich, wie Henk ten Have und Annique Lelie [15] feststellen, als eine Form angewandter Ethik entwickelt, in der allgemeine theoretische Prinzipien auf die Praxis der Medizin und des Gesundheitswesens ,,angewendet“ werden. Diese Kritik richtet sich nicht so sehr an die gängige medizinische Ethik an sich, sondern an eine bestimmte Konzeption der angewandten Ethik. Diese Konzeption einer praktischen Ethik beruht auf einem top-down-Modell und damit auf einem rationalistischen und deduktiven Modell. Die Kritik stimmt mit den Bedenken überein, die von den sogenannten „Antitheoretikern“ gegen die dominante Prinzipienethik vorgebracht werden. ${ }^{9}$ Die Kritiker der dominierenden medizinischen Ethik befürworten daher eine praxisorientierte Wende. In ihren Augen impliziert diese Wende zur Praxis, dass die medizinische Ethik empirischer werden muss.

\footnotetext{
${ }^{8}$ Robert Zussman kommt zu einer ähnlichen Feststellung. Die Verantwortung für eine Reanimationsentscheidung könne nicht alleine den Ärzten oder nur der Familie angelastet werden. Beide zögen die Argumente des anderen in Erwägung: "Decisions were considered and reconsidered, made and remade, negotiated and renegotiated. Decisions were not an event but a process" ([40], S. 159).

Ein anderer Autor, der darauf hinweist, dass Bioethiker es verabsäumen, sozialwissenschaftliche Studien und das Umfeld sowie den institutionellen Kontext und die kulturellen Kräfte (ausreichend) zur Kenntnis zu nehmen, welche die von ihnen bearbeiteten Fragen beeinflussen, ist Bruce Jennings. Bioethiker gingen nach wie vor von kühnen Annahmen über Umfeld, Kontext und Kultur aus: "These claims, in turn, are not harmless asides, sociological obiter dicta; they do affect the normative ethical position offered in various ways. For one thing, these sociological claims affect the reasonableness and persuasiveness of the arguments made, since the reasonableness of an ethical argument that balances various conflicting values and weighs benefits often depends on the context and setting within which the balancing and weighing goes on" ([16], S.262).

${ }^{9}$ Für die Kritik der Antitheoretiker siehe u.a. Cheryl Noble [24], Annette Baier [1] und S.R.L. Clarke [6]. Interne Kritiker der dominanten medizinischen Ethik sind u. a. die niederländischen Medizinethiker Henk ten Have und Annique Lelie [15] sowie Lieke van der Scheer und Guy Widdershoven [28, 39]. Ihr Bezugssystem ist vor allem die Aristotelische Ethik; des Weiteren bei Widdershoven auch Gadamers Hermeneutik und bei Van der Scheer der Pragmatismus von Dewey.
} 
Die allgemeine Zielsetzung der empirischen Ethik möchte ich als die Förderung der Kontextsensitivität der Ethik beschreiben. Die empirisch-ethische Forschung selbst hat keine eigene Methodologie. Obwohl die eingesetzte sozialwissenschaftliche Forschung meist qualitativer Natur ist, kann die empirisch-ethische Forschung genauso gut quantitative Methoden verwenden. Was die Methode der philosophischen Forschung betrifft, so werden hier empirisch-ethische Untersuchungen sowohl aus analytischer als auch aus pragmatischhermeneutischer Sicht durchgeführt. ${ }^{10}$

\section{Generalistische und kontextualistische empirische Ethik}

Es gibt viel mehr Untersuchungen, die den Charakteristika der empirischen Ethik entsprechen, als Untersuchungen, die sich explizit als empirisch-ethisch deklarieren. Ich unterscheide deshalb zuerst zwischen impliziter und expliziter empirischer Ethik. Weiterhin unterscheide ich zwischen zwei Richtungen innerhalb der empirischen Ethik, die ich als generalistische und als kontextualistische empirische Ethik bezeichne. ${ }^{11}$ Die Kontroverse zwischen Generalisten und Kontextualisten (zu denen ich die Partikularisten und die Kohärentisten zähle) findet sich nämlich auch innerhalb der empirischen Ethik. Die Generalisten sind der Ansicht, dass Prinzipien und Vorschriften die Grundlage der Moral bilden. Aus der Sicht der Kontextualisten ist Moral in sozialen Praktiken verankert. In diesem Ansatz ist daher sozialwissenschaftlich-empirisches Vorgehen notwendig, um Moral zu entdecken, während es die Aufgabe der Philosophie ist, sie zu artikulieren und zu interpretieren. Die partikularistische Variante der kontextualistischen empirischen Ethik geht von der Priorität konkreter Urteile der Handelnden aus und funktioniert somit bottom-up, während die kohärentistische Variante weder den konkreten Urteilen noch den ethischen Theorien und Prinzipien Priorität einräumt. Anschließend will ich für beide Hauptrichtungen ein Beispiel anführen. Es handelt sich um drei Beispiele explizit empirisch-ethischer Forschung, die von den Forschern selbst auch als solche deklariert wird. Alle drei beziehen sich auf den Respekt gegenüber der Patientenautonomie. Dies ist im Hinblick auf die beiden niederländischen Studien ist nicht zufällig, denn Autonomie war ein vorrangiges Thema im Forschungsprogramm „Ethik und Politik“ der „Nederlandse Organisatie voor Wetenschappelijk Onderzoek, NWO“ [Niederländische Forschungsorganisation].

Ich beginne mit der Studie der niederländischen Medizinethikerin Maartje Schermer [29], welche ich zu den Generalisten zähle. Ziel dieser Studie war es, die ethische Theorie hinsichtlich der Patientenautonomie weiterzuentwickeln und zu verfeinern, und zwar durch eine Konzeption von Patientenautonomie und ihrer Respektierung, die für die Krankenhauspraxis relevant sein soll. Sie führte umfangreiche Analysen von zentralen Begriffen wie Autonomie, Paternalismus, informierte Zustimmung und Entscheidungsfähigkeit (Kompetenz) durch. Ihre empirische Untersuchung war qualitativer Art und umfasste eine Anzahl von Interviews mit älteren Krankenhauspatienten (über 65 Jahre). Ziel war es, Fallberichte zu sammeln, welche ausreichend heuristisches Potenzial hatten, um zu verdeutlichen, was Patientenautonomie in der Krankenhauspraxis tatsächlich beinhaltet, und auf diese Weise zur weiteren Entwicklung der ethischen Theorie der Patientenautonomie beizutragen. Ihrer Aussage nach hat die empirische Studie Folgendes ergeben: 1) Die Fälle machten deutlich, welche normativen und praktischen Probleme verschiedene, bestehende theoretische

\footnotetext{
${ }^{10}$ Beide Betrachtungsweisen sind im Band von Widdershoven et al. [38] vertreten.

11 An anderer Stelle qualifiziere ich die generalistische empirische Ethik als schwache und die kontextualistische empirische Ethik als starke empirische Ethik [22].
} 
Ansätze zur Patientenautonomie mit sich bringen. 2) Die Fälle lenkten die Aufmerksamkeit auf Fragen und Themen, die in der ethischen Theorie nur wenig Aufmerksamkeit erhalten hatten, wie zum Beispiel Vertrauen. 3) Darüber hinaus suggerierten die Fälle, wie bestimmte Fragen und Probleme am besten angegangen werden können.

Die zweite Studie, durchgeführt von den niederländischen Medizinethikern Ghislaine van Thiel und Hans van Delden [34], ist ein Beispiel für kontextualistische empirisch-ethische Forschung (kohärentistische Variante). Die Studie betrifft ebenfalls den Umgang mit Autonomie, diesmal jedoch im Kontext des Pflegeheims. Die zentrale Frage dieser Studie ist, ob es wünschenswert und möglich ist, in Pflegeheimen die Respektierung der Autonomie in die Praxis umzusetzen, wobei darunter die Respektierung der Selbstbestimmung, der Mitentscheidung, die Respektierung der Privatsphäre und der eigenen Normen und Werte [der Patienten] verstanden wird. Die Forscher erfassten die moralischen Intuitionen des Pflegepersonals hinsichtlich der Respektierung der Autonomie mit der Frage, welche von vier Pflegeauffassungen, von denen jede auf einer spezifischen Autonomievorstellung beruhte (liberal, kantianisch, biographisch und pflegeethisch), sie für den Kontext eines Pflegeheims am besten geeignet fanden. Pflegepersonen, die mit somatischen Patienten arbeiteten, entschieden sich relativ häufiger für die kantianische Sicht, während Pflegepersonen von psychogeriatrischen Patienten mehr zur biographischen (narrativen) Vorgangsweise neigten. Die Schlussfolgerung war, dass eine einzige Auffassung von Autonomie für sich alleine nicht in allen Situationen dem am besten gerecht wird, was Pflegepersonen für ihr Handeln als moralisch richtig ansehen.

Die dritte Studie, durchgeführt von den englischen Medizinethikern Jacintha Tan und Tony Hope [32], zähle ich zur partikularistischen Variante der kontextualistischen empirischen Ethik. Es geht hierbei um die Frage, ob eine Zwangsbehandlung von Patienten mit Anorexia nervosa gerechtfertigt ist. Zur Beantwortung dieser Frage muss man wissen, ob diese Patienten entscheidungsfähig (kompetent) sind. Die Forscher interviewten eine Anzahl von Anorexiepatienten und führten mit ihnen auch einen standardisierten Kompetenztest durch. Es zeigte sich, dass die Patienten im Kompetenztest gute Werte erreichten und somit hinsichtlich der Behandlungsverweigerung als kompetent gelten mussten. Die Interviews hingegen zeigten, dass die Patienten zwar einsahen, dass andere Menschen in einer vergleichbaren Situation große Risiken eingehen würden, wenn sie sich nicht behandeln ließen. Tief im Inneren glaubten sie aber, selbst kein signifikantes Risiko einzugehen, da sie sich in keinerlei Hinsicht krank oder abnormal fühlten. Ihre Überzeugungen waren offenbar das Resultat „emotionaler Argumentation“. Die Forscher fragten sich, ob und unter welchen Voraussetzungen die Anorexie als wesentlicher Bestandteil der Identität einer Person angesehen werden kann und ob dieses Faktum für die Frage nach der Rechtfertigung einer Zwangsbehandlung relevant ist. Sie kamen zu der Schlussfolgerung, dass bestehende Konzepte zur Fähigkeit zur Behandlungsverweigerung und zu deren Implikationen sowohl für die Theorie als auch für die Praxis erheblich weiterentwickelt werden müssen, um den empirischen Ergebnissen gerecht zu werden.

\section{Probleme der empirischen Ethik}

Im Rahmen dieses Artikels ist es nicht möglich, allen Fragen, welche die empirische Ethik aufwirft, gerecht zu werden. Ich beschränke mich daher darauf, für jede der Hauptströmungen der empirischen Ethik je eine kritische Anfrage zur Sprache zu bringen. Bevor ich damit beginne, eine kurze Bemerkung zu der Frage, ob die empirische Ethik sich des naturalistischen Fehlschlusses schuldig macht oder die Kluft zwischen is und ought auf 
illegitime Weise überbrückt. Kürzlich haben die niederländischen Ethiker Bert Gordijn und Rob de Vries meiner Meinung nach überzeugend dargelegt, dass hiervon in der empirischen Ethik, wie sie oben beschrieben wurde, keine Rede ist [14]. Was man auch von der Trennung zwischen Fakten und Werten und der Kluft zwischen „,sein“ und „sollen“ halten mag, die empirische Ethik hebt diese nicht auf. Wie eng die ethische Analyse und die empirische Forschung in der empirischen Ethik auch miteinander verflochten sein mögen, die Eigenart und die eigene Methodologie beider können (und müssen) erhalten bleiben (vgl. auch andere Beiträge in diesem Heft).

Nun zu meiner Frage zur generalistischen empirischen Ethik: Dieter Birnbacher hält als Utilitarist und Generalist in einem Artikel aus dem Jahre 1999 ein Plädoyer für die Zusammenarbeit zwischen praktischer Ethik und Sozialwissenschaften. Am Anfang des Artikels behauptet er, dass es zwei Gründe dafür gibt, in der praktischen Ethik der Empirie Aufmerksamkeit zu schenken. Der erste Grund gilt sowohl für den Ethiker als auch für den Juristen:

As the jurist's task is not only to make sure that a particular proposal of legislation is compatible with constitutional norms and the general principles recognised in the system of law concerned, but also to look to the practicability and effectiveness of the proposed piece of legislation (given its aim), the applied ethicist has the same dual responsibility. His role is not only to inquire into theoretical merits of a proposed norm of practical morality (in terms of internal consistency, coherence with other rules of social morality, and compatibility with underlying principles) but also to consider its practical feasibility, its psychological acceptability and its potential effectiveness in changing the attitudes and behaviour in the desired direction. ([3], S. 321)

Ein zweiter Grund, in der praktischen Ethik empirische Daten einzubeziehen, ist nach Birnbacher "relevant to all those approaches which conceive of applied ethics as the 'application', literally understood, of theoretical principles to real-life cases via maxims, middlerange principles or practice rules. According to this conception, applied ethics deals with the 'translation', as it were, of theoretical principles into workable practice rules, making them available for everyday judgements and decisions" ([3], S. 321). Birnbacher formuliert anschließend in einzelnen Thesen die Anforderungen, denen Praxisregeln genügen müssen. Er führt dabei in einzelnen Fällen auch an, welche empirische Daten notwendig sind, um die Regeln darauf abzustimmen. Eine der Thesen Birnbachers besagt, dass Praxisregeln, um auch tatsächlich dem Handeln eine Richtung geben zu können, so formuliert sein müssen, dass sie mit bereits vorhandenen, traditionellen moralischen Überzeugungen in Einklang zu bringen sind ([3], S. 325). Eine andere These besagt, dass Praxisregeln nicht zu viel, aber auch nicht zu wenig fordern dürfen. Ohne von bestimmten psychologischen Annahmen auszugehen sei es jedoch nicht möglich, das Ausmaß zu bestimmen, in dem man erwarten könne, dass die Regeln auch tatsächlich zu entsprechendem Verhalten und entsprechenden Haltungen führten ([3], S. 325). Birnbacher geht es letztendlich darum, die Durchführbarkeit und Realisierbarkeit von Praxisregeln als Übersetzung ethischer Prinzipien zu fördern. Die Daten, die nach Birnbacher notwendig sind, um Prinzipien in Praxisregeln zu übersetzen, zählen nicht ausschließlich zum Bereich der Soziologie, sondern auch zu jenem der Psychologie. Das Problem ist allerdings, dass die Durchführbarkeit und Realisierbarkeit sowohl von den objektiven als auch subjektiven Eigenschaften von jenen Personen abhängen, für welche die Regeln bestimmt sind. Wenn jemand eine bestimmte Steuer nicht bezahlen kann, weil er dann nicht genug Geld für seine primären Lebensbedürfnisse übrig hat, dann ist das ein mehr oder weniger objektives Faktum. Es kann aber sein, dass jemand nicht motiviert ist, die Steuer zu bezahlen, weil er dem Ziel, für welches das Steuergeld 
verwendet wird, nicht zustimmt. Die Frage ist, wieweit man bei der Formulierung von Praxisregeln einen inhaltlich motivierten Widerstand gegen das berücksichtigen muss, was die Regeln vorschreiben.

Die kritische Frage, die ich hinsichtlich der kontextualistischen empirischen Ethik zur Sprache bringen will, hängt mit ihrem Ausgangspunkt zusammen, nämlich damit, dass Moral in sozialen Praktiken verwirklicht ist. Durch diesen Ausgangspunkt ist die kontextualistische empirische Ethik ihrem Wesen nach auf die Zusammenarbeit mit den Sozialwissenschaften angewiesen. Diese untersuchen die moralische Erfahrung derer, welche die Praktiken ausüben, da diese wiederum den Zugang zur Moral dieser Praktiken bildet. Dies kommt in der Beschreibung der Grundvoraussetzungen der empirischen Ethik von Borry et al. klar zum Ausdruck:

Empirical ethics is a broad category, grasping different interpretations of combining or trying to integrate ethics and empirical research. [...] Although there are various ways of combining empirical research and ethical reflection (and of doing empirical ethics), they all have some basic assumptions in common: firstly, empirical ethics states that the study of people's actual beliefs, intuitions, behaviour and reasoning yields information that is meaningful for ethics and should be the starting point of ethics, secondly, empirical ethics acknowledges that the methodology of the social sciences (with quantitative and qualitative methods such as case studies, surveys, experiments, interviews, and participatory observation) is a way (and probably the best way) to map this reality; [...] ([4], S. 1).

Nach Ansicht von Borry et al. benötigt die empirische Ethik die Sozialwissenschaften, um die tatsächlichen moralischen Überzeugungen, Intuitionen, Verhaltensweisen und Argumente zu untersuchen, welche den Ausgangspunkt der Ethik bilden müssen. Es ist jedoch nicht klar, ob Borry und seine Kollegen bei einer derartigen Studie den Meinungen der Ausübenden einer Praxis besondere Aufmerksamkeit schenken wollen. Dies ist bei den niederländischen kohärentistischen Medizinethikern Ghislaine van Thiel und Hans van Delden sehr wohl der Fall. Sie behaupten, dass vor allem die Intuitionen von Menschen, die in einer Praxis leben und arbeiten, in ein Reflexionsmodell moralischer Argumentation und Aussagen eingebracht werden müssen, da sie eine „spezifische moralische Weisheit“ besitzen [35]. Ihre partikularistischen niederländischen Kollegen Guy Widdershoven und Lieke van de Scheer sind noch expliziter: "If practice is seen as a source for ethics, the experience, insight, and arguments of practitioners form the departure for philosophical ethics" ([39], S. 25). Das heißt jedoch nicht, dass die Berücksichtigung der moralischen Überzeugungen der Ausübenden einer Praxis ein spezifisches Merkmal der kontextualistischen empirischen Ethik ist. Auch im Rahmen einer generalistischen empirischen Ethik gibt es Gründe dafür, sich einen Überblick über deren moralische Überzeugungen zu verschaffen und sie ernst zu nehmen. Eine der Thesen Birnbachers besagt, dass Praxisregeln, um tatsächlich dem Handeln eine Richtung geben zu können, so formuliert werden müssen, dass der Nachdruck auf deren Kontinuität mit bereits vorhandenen, traditionellen moralischen Überzeugungen liegt ([3], S. 325). Es ist allerdings ein spezifisches Merkmal der kontextualistischen empirischen Ethik, dass den moralischen Intuitionen und Überzeugungen von Ausübenden einer Praxis ein spezieller epistemischer Status zuerkannt wird. Meiner Meinung nach gibt es für diesen speziellen Status keine hinreichende Rechtfertigung. Dadurch kommt es insbesondere bei der partikularistischen Variante der kontextualistischen empirischen Ethik zu Problemen.

Die Idee, dass die Moral in Praktiken verankert ist, stammt von Alasdair MacIntyre. Er definiert Praxis als eine "coherent and complex form of socially established cooperative human activity" ([18], S. 187). Eine Praxis sei eine Aktivität, in der bestimmte interne Güter 
realisiert werden. Interne Güter verkörperten die wesentlichsten qualitativen Ingredienzien von Praktiken. Interne Güter seien von externen Gütern zu unterscheiden, die mit einer Praxis nur kontingent verbunden seien. Interne Güter würden realisiert, wenn man versuche, den Standards der Vorzüglichkeit, die dieser Aktivität eigen seien, zu entsprechen. Um in einer Praxis in vollem Maße und erfolgreich agieren zu können, müsse man bestimmte Charaktereigenschaften oder Tugenden erwerben. Die amerikanischen Ethiker Edmund Pellegrino und David Thomasma entwickelten auf der Grundlage der Ideen von MacIntyre eine tugendethische Medizinethik. Ihrer Ansicht nach impliziert die Medizin als menschliche Aktivität die Annahme bestimmter interner Güter und moralischer Standards. Dies seien „moralische Imperative“, die eine "internal morality of medicine - something built into the nature of medicine as a particular kind of human activity" konstituierten ([26], S. 42). Die interne Moral der Medizin muss von ihrer externen Moral unterschieden werden, die von Henk ten Have und Annique Lelie - kontextualistisch-empirische Ethiker - definiert wird als "values, norms and rules prevailing in social, cultural and religious traditions that function as external determinants of medicine" ([15], S. 268). Nach MacIntyre können interne Güter nur durch erfahrene, kompetente Ausübende einer Praxis vollständig erkannt und identifiziert werden. Eine ähnliche Sicht vertreten auch die kontextualistisch-empirischen Ethiker hinsichtlich der internen Moral einer Praxis. Stimmt diese Sicht, dann wäre es auch gerechtfertigt, den moralischen Intuitionen und Überzeugungen der Ausübenden einer Praxis eine Art epistemisches Privileg zuzuerkennen.

Ich bezweifle jedoch, dass die Ansicht von MacIntyre, interne Güter würden nur durch erfahrene, kompetente Ausübende einer Praxis vollständig erkannt und identifiziert, auch auf die interne Moral einer Praxis wie der Medizin anwendbar ist. MacIntyres Sicht ist epistemologischer Natur. Ich sehe ein, dass ich nicht imstande bin, die internen Güter des Komponierens von Musik zu identifizieren und zu erkennen. Ich bin weder mit dieser noch mit einer ähnlichen Praxis vertraut. Ich bin nicht in einem Beruf des Gesundheitswesens beschäftigt, ich kenne jedoch andere Pflegepraktiken. Ich nehme deshalb an, dass ich in gewissem Ausmaß in der Lage bin, die internen Güter der Medizin und der Pflege zu erkennen und zu identifizieren. Ein weiteres Argument für meine Behauptung, dass MacIntyres These auf die interne Moral der Medizin nicht zutrifft, ist, dass die Konzeption der internen Moral nicht nur interne Güter der Medizin umfasst, sondern auch Normen und Werte für den Umgang zwischen Arzt und Patient. Die amerikanischen Ethiker Frank Miller und Howard Brody sind der Ansicht, dass die Ziele der Medizin nicht statisch sind, sondern sich ständig weiterentwickeln. Die traditionellen Ziele und Pflichten von Ärzten würden neu interpretiert, und gleichzeitig würden auch neue Ziele und Pflichten erkannt ([20], S. 585 ff.). Die fortschreitende Debatte über die Ziele der Medizin und die Pflichten von Ärzten sei übrigens niemals nur eine Angelegenheit der Ärzte gewesen. Die Geschichte der medizinischen Ethik lasse auch erkennen, dass Einflüsse von außen Veränderungen der inneren Moral der Medizin in Gang setzen könnten ([2], S. 606; [5], S. 397; [20], S. 585). Der bereits genannte Robert Zussman merkt zu Recht an, dass die heutige medizinische Ethik aus einem Set von Werten entstanden ist, die, wie z.B. die Selbstbestimmung, historisch gesehen den medizinischen Traditionen fremd sind.

Es gibt noch mehr Argumente, die dagegen sprechen, den Ausübenden einer Praxis ein epistemisches Privileg zuzuerkennen, die ich jedoch im Rahmen dieses Artikels nicht besprechen kann. ${ }^{12}$ Wenn ich recht habe, so ist es falsch, davon auszugehen, dass jene, die in einer Praxis leben und arbeiten, aufgrund ihrer Erfahrung besser als Außenstehende imstande sind, die interne Moral der Medizin zu erkennen und zu identifizieren. Diese Schlussfolge-

12 Ich tue dies in [23]. 
rung hat Konsequenzen für die Planung empirisch-ethischer Studien, insbesondere für die Auswahl der Studienteilnehmer.

\section{Zusammenfassende Bewertung}

Alle drei genannten Studien beinhalten relevante Erkenntnisse. Besonders die letzte Studie sollte Anlass zum Nachdenken geben. Derartige Beispiele überzeugen mich, dass eine empirische Ethik, welche ethische Analyse und empirische Forschung in einem interaktiven Zyklus kombiniert, sinnvoll ist. Die empirische Ethik nimmt die Intention der praktischen Ethik ernst, nämlich ihr Bestreben, nicht nur Vorschriften zu machen, sondern das Handeln von Menschen auch tatsächlich anzuleiten. Die empirische Ethik betrachtet das Bemühen um Kontextsensitivität moralischer Ratschläge, Leitlinien und Prinzipien nicht als Teil eines Nachfolgeprozesses, für den andere als Ethiker verantwortlich sind, sondern als Teil eines Hauptprozesses, für den sie selbst verantwortlich ist.

Obwohl ich den Aufstieg der empirischen Ethik insgesamt als positiv bewerte, möchte ich trotzdem einige kritische Anmerkungen zu ihrer Entwicklung machen. Die erste Anmerkung ist, dass ich mir über den normativen und philosophischen Gehalt empirisch-ethischer Studien Sorgen mache. Soll die empirisch-ethische Forschung dem Ideal des interaktiven Zyklus von McMillan und Hope gerecht werden, muss der Forscher sowohl sozialwissenschaftlich als auch philosophisch kompetent sein, oder, wenn es sich um mehrere Forscher handelt, muss zumindest einer von ihnen über diese Kompetenzen verfügen. Diese Forderung wird bei weitem nicht immer erfüllt. ${ }^{13}$ Weiterhin besteht die Gefahr, dass, wenn für eine Untersuchung nur begrenzte Zeit verfügbar ist, der philosophische Gesichtswinkel am ehesten unterbelichtet bleibt, da es durch den höheren Standardisierungsgrad eher auffällt, wenn der empirische Teil der Studie nicht den methodischen Anforderungen entspricht. Die zweite Anmerkung ist eher allgemeiner Art: Ich würde es sehr bedauern, wenn die gesamte Forschung auf dem Gebiet der Medizinethik empirisch-ethisch werden sollte. Es muss genügend Raum für die traditionelle philosophisch-ethische Forschung bleiben, die zwar empirisch gut informiert ist, selbst aber nicht mit empirischer Forschung verbunden ist. Ein gutes Beispiel ist meiner Meinung nach Human Identity and Bioethics von David DeGrazia [7]. Leider ist es zumindest in den Niederlanden so, dass Medizinethiker in ihrer Publikationsstrategie den Impact Factor von Zeitschriften berücksichtigen müssen. In der Medizin haben philosophische Zeitschriften auf dem Gebiet der Medizinethik einen niedrigen Impact Factor. Traditionell-philosophische medizinethische Publikationen lohnen sich zu wenig, jedenfalls für Medizinethiker an medizinischen Fakultäten.

Open Access Dieser Artikel unterliegt den Bedingungen der Creative Commons Attribution Noncommercial License. Dadurch sind die nichtkommerzielle Nutzung, Verteilung und Reproduktion erlaubt, sofern der/die Originalautor/en und die Quelle angegeben sind.

\section{Literatur}

1. Baier A (1985) Doing without moral theory. In: Baier A (ed) Postures of the mind. Essays on mind and morals. Methuen, London, pp 228-245

2. Beauchamp TL (2001) Internal and external standards for medical morality. J Med Phil 36:601-619

\footnotetext{
${ }^{13}$ Ich stütze mich hier auf meine Erfahrungen als Mitglied des Lenkungsausschusses des NWO-Programms „Ethik und Politik“ und als Mitglied der Programmkommission seines Nachfolgeprogramms, des NWO-Programms „Ethik, Forschung und Führung“.
} 
3. Birnbacher D (1999) Ethics and social science: Which kind of co-operation? Eth Th Mor Pr 2:319-336

4. Borry P, Schotsman P, Dierickx K (2004) Editorial. Empirical ethics: A challenge to bioethics. Med Health Care Phil 7:1-3

5. Brody H, Miller FG (1998) The internal morality of medicine: Explication and application to managed care. J Med Phil 23:384-410

6. Clarke SRL (1989) Abstract morality, concrete cases. In: Evans JDG (ed) Moral philosophy and contemporary problems. Cambridge University Press, Cambridge, pp 35-54

7. DeGrazia D (2005) Human identity and bioethics. Cambridge University Press, Cambridge

8. Doris JM (2002) Lack of character. Cambridge University Press, Cambridge

9. Edel A (1955) Ethical judgment. The Free Press, Glencoe IL

10. Edel A (1961) Science and the structure of ethics. University of Chicago Press, Chicago

11. Feibleman JK (1955) Introduction to an objective, empirical ethics. Ethics 56:102-115

12. Foot Ph (2001) Natural goodness. Oxford University Press, Oxford

13. Fox R (1989) The sociology of medicine: A participant observer's view. Prentice Hall, Englewood Cliffs

14. Gordijn B, Vries R de (2009) Empirical ethics and its alleged naturalistic fallacies. Bioethics 23:193-201

15. Have HAJM ten, Lelie A (1998) Medical ethics research between theory and practice. Theor Med Bioethics 19:263-276

16. Jennings B (1990) Ethics and ethnography in neonatal intensive care. In: Weisz G (ed) Social science perspectives on medical ethics. Kluwer Academic Publishers, Dordrecht, pp 261-270

17. Kruithof J (1973) Eticologie. Inleiding tot de studie van het morele verschijnsel. Boom, Meppel

18. MacIntyre A (1981) After virtue: A study in moral theory. University of Notre Dame Press, Notre Dame

19. McMillan J, Hope T (2008) The possibility of empirical psychiatric ethics. In: Widdershoven G, McMillan J, Hope T, Scheer L van der (eds) Empirical ethics in psychiatry. Oxford University Press, Oxford, pp 9-23

20. Miller FG, Brody H (2001) The internal morality of medicine: An evolutionary perspective. J Med Phil 26:581-599

21. Molewijk B, Stiggelbout A, Otten W, Dupuis HM, Kievit F (2004) Empirical data and moral theory. A plea for integrated empirical ethics. Med Health Care Phil 7:55-69

22. Musschenga AW (2005) Empirical ethics, context-sensitivity and contextualism. J Phil Med 30:1-24

23. Musschenga AW (2010) Empirical ethics and the special status of the practitioners' judgements. In: Draulans V et al (eds) The sensitive relation between empirical sciences and ethics (provisional title). Peeters, Leuven (in press)

24. Noble C (1979) Normative ethical theories. The Monist 62:456-509

25. Parker M (2009) Two concepts of empirical ethics. Bioethics 23:202-213

26. Pellegrino ED, Thomasma DC (1993) The virtues in medical practice. Oxford University Press, New York

27. Pool R (1996) Vragen om te sterven. Euthanasie in een Nederlands ziekenhuis. WYT-Uitgeefgroep, Rotterdam

28. Scheer L van der, Widdershoven G (2004) Integrated empirical ethics. Loss of normativity? Med Health Care Phil 7: 71-79

29. Schermer M (2001) The different faces of autonomy: A study on patient autonomy in ethical theory and hospital practice. Doctoral dissertation, Universität Amsterdam, Amsterdam

30. Schmidt V (2000) Bedingte Gerechtigkeit. Soziologische Analysen und Philosophische Theorien. Campus Verlag, Frankfurt

31. Stich S, Doris JM (2007) Empirical perspectives on ethics. In: Jackson F, Smith M (eds) The Oxford handbook of contemporary philosophy, Chap. 5. Oxford University Press, Oxford

32. Tan J, Hope T (2008) Treatment refusal in Anorexia Nervosa. A challenge to current concepts of capacity. In: Widdershoven G, McMillan J, Hope T, Scheer L van der (eds) Empirical ethics in psychiatry. Oxford University Press, Oxford, pp 187-211

33. The A-M (1997) Vanavond om 8 uur: Verpleegkundige dilemma's bij euthanasie en andere beslissingen rond het levenseinde. Bohn StafleuVan Loghum, Houten Diegem

34. Thiel GJMW, Delden JJM van (1997) Dealing with patient autonomy in Dutch nursing homes. Health Care Later Life 3:177-186

35. Thiel GJMW, Delden JJM van (2009) The justificatory power of moral experience. J Med Ethics $35: 234-237$

36. Vivas E (1938) Force in empirical ethics. Ethics 49:85-92 
37. Weaver GR, Trevino LK (1994) Normative and empirical business ethics. Bus Ethics Q 4:129-143

38. Widdershoven G, McMillan J, Hope T, Scheer L van der (eds) (2008) Empirical ethics in psychiatry. Oxford University Press, Oxford

39. Widdershoven G, Scheer L van der (2008) Theory and methodology of empirical ethics: A pragmatichermeneutic perspective. In: Widdershoven G, McMillan J, Hope T, Scheer L van der (eds) Empirical ethics in psychiatry. Oxford University Press, Oxford, pp 23-37

40. Zussman R (1992) Intensive care: Medical ethics and the medical profession. The University of Chicago Press, Chicago 